\title{
On extrapolation of virial coefficients of hard spheres*
}

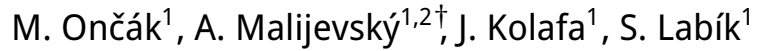 \\ ${ }^{1}$ Institute of Chemical Technology, Prague, Department of Physical Chemistry, 16628 Praha 6, Czech Republic \\ 2 Department of Physics, Faculty of Science, University of Ostrava, 70103 Ostrava 1, Czech Republic
}

Received January 31, 2012, in final form April 18, 2012

\begin{abstract}
Several methods of extrapolating the virial coefficients, including those proposed in this work, are discussed. The methods are demonstrated on predicting higher virial coefficients of one-component hard spheres. Estimated values of the eleventh to fifteenth virial coefficients are suggested. It has been speculated that the virial coefficients, $B_{n}$, beyond $B_{14}$ may decrease with increasing $n$, and may reach negative values at large $n$. The extrapolation techniques may be utilized in other fields of science where the art of extrapolation plays a role.
\end{abstract}

Key words: hard spheres, virial coefficients, extrapolation methods

PACS: 02.30.-f, $51.30 .+i$

\section{Introduction}

Virial coefficients play a key role in both molecular and phenomenological theory of fluids at low and medium densities. They are the coefficients in the density expansion of the equation of state expressed via the compressibility factor

$$
Z=\frac{\beta P}{\rho}=1+\sum_{i=2}^{\infty} B_{i} \rho^{i-1}
$$

where $\beta=1 /\left(k_{\mathrm{B}} T\right)$ is the inverse temperature, $P$ is the pressure, and $\rho$ is the number density. Virial coefficients $B_{i}$ are defined by exact formulae in terms of integrals whose integrands are products of Mayer functions [1].

In this paper we concentrate on the simplest non-trivial model of fluid - the system of hard spheres. For hard spheres, i.e., hard body one-component fluids, the virial coefficients are numbers (they do not depend on temperature). For the system considered, the terms up to $B_{4}$ are known analytically [2, 3]. It holds

$$
\begin{aligned}
& B_{1}=1 \\
& B_{2}=4 \\
& B_{3}=10 \\
& B_{4}=\frac{2707 \pi+438 \sqrt{2}-4131 \arccos (1 / 3)}{70 \pi}=18.364768 \ldots
\end{aligned}
$$

The values are in the units of the packing fraction $\eta=\frac{\pi}{6} \sigma^{3} \rho$ with $\sigma$ standing for the sphere diameter.

The higher virial coefficients must be calculated numerically. Virial coefficients are sums of irreducible (cluster) integrals represented by diagrams [1]. It is a task for a high school student to determine the second virial coefficient for hard spheres. However, dimensionality of irreducible integrals increases rapidly with increasing order of the virial coefficient. In three dimensional space it is $3 n-6$. For example the tenth virial coefficient contains 24-dimensional integrals (!) in the simplest case of spherically symmetric interactions between molecules.

\footnotetext{
${ }^{*}$ Dedicated to Professor Orest A. Pizio on the occasion of his 60th birthday

${ }^{\dagger}$ E-mail: anatol.malijevsky@vscht.cz
} 
Table 1. Number of unlabeled and labeled Mayer and Ree-Hoover diagrams, and dimensionality $d$ of corresponding cluster integrals for hard spheres.

\begin{tabular}{|r|r|r|r|r|r|}
\hline$n$ & \multicolumn{2}{|c|}{ Mayer } & \multicolumn{2}{|c|}{ Ree-Hoover } & \multirow{2}{*}{$d$} \\
\cline { 2 - 5 } & unlabeled & labeled & unlabeled & labeled & \\
\hline \hline 2 & 1 & 1 & 1 & 1 & 1 \\
3 & 1 & 1 & 1 & 1 & 3 \\
4 & 3 & 10 & 2 & 4 & 6 \\
5 & 10 & 238 & 5 & 68 & 9 \\
6 & 56 & 11368 & 23 & 3053 & 12 \\
7 & 468 & 1014888 & 171 & 297171 & 15 \\
8 & 7123 & 166537616 & 2606 & 56671216 & 18 \\
9 & 194066 & 50680432112 & 81564 & 21286987064 & 21 \\
\hline
\end{tabular}

Numbers of integrals (no general formula is known for the number of irreducible diagrams in dependence on $n$ ) increase even much more rapidly than the dimensionality of the integrals, see table 1 . Some of them are topologically equivalent which means that their integrands differ only in the numbering of variables. In the table, the heading "labeled" denotes the total number of irreducible diagrams and "unlabeled" denotes the number of topologically different diagrams with numbered black points. To reduce these numbers, Ree and Hoover in their pioneering work [4] replaced Mayer diagrams with $f$ bonds by generalized Ree-Hoover diagrams with $f$-bonds and $e$-bonds using the identity $f(r)+e(r)=1$. Besides the reduction of the number of topologically different diagrams, there is another advantage of the Ree-Hoover approach: computer codes using these diagrams are more efficient than the Mayer approach.

For higher virial coefficients, say $n>7$, it is impossible within an average lifetime of an explorer to determine the diagrams and their weights using "pencil and paper" avoiding "human factor" errors. For example, for $B_{8}$ there are 7123 different Mayer diagrams which can be reduced to 2606 Ree-Hover diagrams, and for each of them their weights should be determined. The analysis should be done automatically on a computer. Different algorithms utilizing symbolic algebra programming and automatic code generation have been proposed [5-8].

Another problem is to evaluate the multi-fold integrals. This can be done by random-shooting Monte Carlo integration but it may require too much computer time. A more efficient way is to start from the so-called spanning diagrams - the integrals that can be calculated analytically. Configurations of the spanning diagrams are sampled either using a standard Metropolis Monte Carlo method or (for the linear chains) by the so-called reptation [9]. The values of the diagrams of interest are then evaluated by Monte Carlo integration.

Table 2. Summary of recommended virial coefficients $B_{n}$ for hard spheres. Values in parentheses are standard errors in the last significant digit.

\begin{tabular}{|c|c|}
\hline$n$ & $B_{n}$ \\
\hline \hline 5 & $28.22445(10)$ \\
6 & $39.81545(34)$ \\
7 & $53.3418(15)$ \\
8 & $68.5394(87)$ \\
9 & $85.805(58)$ \\
10 & $105.8(4)$ \\
\hline
\end{tabular}

The fifth virial coefficient for hard spheres was calculated by Rosenbluth and Rosenbluth [10] and by Kratky [11-14], the sixth by Ree and Hoover [4], the seventh by Ree and Hoover [15], by Kim and 
Henderson [16], and by Janse van Rensburg and Torrie [17], the eighth by Janse van Rensburg [18] and by Vlasov et al. [5], the ninth by Labík et al. [6], and the tenth by Clisby and McCoy [7, 8]. As a rule, while higher virial coefficients were just calculated, the lower ones were more precisely recalculated. The stateof-the-art values of the virial coefficients for hard spheres are shown in table22including their estimated uncertainties.

\section{Results and discussion}

\subsection{Extrapolation of Virial Coefficients}

It is prohibitively difficult to calculate virial coefficients beyond $B_{10}$ because the numbers of diagrams increase enormously. There are $2^{\left(\begin{array}{c}n \\ 2\end{array}\right)}$ diagrams with $n$ field points that should be analyzed. It is $2^{45}$ for $n=10,2^{55}$ for $n=11,2^{66}$ for $n=12$, etc. The number of diagrams increases by more than three orders with $n$ going from 9 to 10. It is thus practical to estimate higher virial coefficients for $n>10$ rather than to try to calculate them.

There are several approaches to extrapolation of virial coefficients based on an (unjustified) assumption that the higher coefficients depend on the lower ones. In the theory of fluids the most popular are Padé approximants, see [19] and references therein. They are based on the assumption that the compressibility factor may be expressed in the form

$$
Z=\frac{P_{n}(\eta)}{Q_{m}(\eta)},
$$

where $P_{n}$ and $Q_{m}$ are polynomials of the order $n$ and $m$, respectively, whose coefficients are combinations of the known virial coefficients. By expanding equation (2.1) into the Taylor series, higher virial coefficients are obtained.

Differential approximants [20] are an extension of Padé approximants. The first order differential approximants have the form

$$
P_{n}(\eta) \frac{\mathrm{d} Z}{\mathrm{~d} \eta}+Q_{m}(\eta) Z(\eta)=R_{m}(\eta),
$$

where $P_{n}, Q_{m}$, and $R_{m}$ are polynomials; for $P_{n}(\eta)=0$, the Padé approximants are revealed. Differential approximants have not been used in the theories of fluids so far.

These two approaches utilize only a part of available information, namely the lower virial coefficients. Another source of information are computer simulation data on the compressibility factors. They can be utilized by proposing an equation of state (EOS) some of its parameters being determined from the known virial coefficients and the remaining constants fitted to the simulation data. For example, Kolafa et al. [21] used an equation of state in the form proposed by Barboy and Gelbart [22]

$$
Z=1+\sum_{i=1}^{n} a_{i} x^{i}, \quad x=\frac{\eta}{1-\eta},
$$

where lower $a_{i}$ are combinations of the known $B_{i}$ and the higher ones are fitted to the computer simulation data. By expanding the equation in powers of $\eta$, the higher virial coefficients may be estimated.

This approach does not take into account the suggested convergence limit at high densities [23]. Therefore, we extended the form (2.3) by changing the pole $\eta=1$ into

$$
x=\frac{\eta}{\eta_{\text {pole }}-\eta},
$$

where $\eta_{\text {pole }}$ is a (nonlinear) adjustable parameter. This pronounced pole should not be confused with a weak nonanalyticity at the freezing point discussed below.

All the traditional extrapolation methods suffer from an assumption that the values of lower virial coefficients are known precisely. This is, certainly, not true, see table 2 To be accurate, uncertainties in the lower virial coefficients (as well as in EOS simulation data) should be taken into account [24]. 
We believe that the safest way of utilizing both the lower virial coefficients data and the EOS data is to minimize the objective function

$$
F=\sum_{i=5}^{n}\left(\frac{B_{i}-B_{i}^{\exp }}{\sigma_{i}}\right)^{2}+\sum_{j=1}^{k}\left(\frac{Z_{j}-Z_{j}^{\exp }}{\sigma_{j}}\right)^{2}
$$

where $n$ is a number of known virial coefficients, $\sigma_{i}$ are their standard deviations, $k$ is the number of simulated state points, and $\sigma_{j}$ are their standard deviations. $Z_{j}$ is the value of the compressibility factor at density $\eta_{j}$ given by a chosen compressibility factor in the form of equation (2.1) (Padé approximants), or in the form of equation (2.2) (differential approximants), or in the form of equation (2.3) (BarboyGelbart equation).

As a measure of fit accuracy we use a standard deviation $S$

$$
S=\sqrt{\frac{F}{n-p}},
$$

where $p$ is a number of adjustable parameters.

\subsection{Barboy-Gelbart approach}

Let us return to equations (2.3) and (2.4). We allow for zero coefficients $a_{i} \mathrm{~s}$ in the expansions. This improves precision without adding more adjustable parameters especially at higher densities. For instance, equation (10) of [21], valid up to $\rho=0.98$, uses powers $x^{1}$ to $x^{8}$ and $x^{12}$. Therefore, the choice of powers serves as another (discrete) adjustable parameter.

In order to elucidate the ability of expansions to predict unknown virial coefficients we repeated the calculations with forms (2.3) and (2.5), using various sets of input data. That is, we "forget" that we know the virial coefficients up to $B_{10}$ and use only a subset to $n, 4 \leqslant n \leqslant 10$.

Moreover, we employ only subsets of available EOS data. For $\rho=0.94$ the error in $B_{11} \pm 1$ propagates to the error in $Z \pm 0.00083$ which is 8 times the standard deviation of the MD datum. The density range lower than $\rho_{\max }<0.94$, would not contain enough information to allow for extrapolating the virial coefficients. On the other hand, the equation of state is nonanalytical at the freezing point (density 0.947) [25], although the nonanalycity term is tiny and in moderately metastable region it is not detectable within available precision. Therefore, we choose $\rho_{\max }=0.98$ as a compromise and calculate all fits with five EOS sets from low densities up to $\rho_{\max }=0.94,0.95,0.96,0.97,0.98$.

Table 3. Barboy-Gelbart predictions of $B_{n}$ using the equation of state up to reduced densities 0.94 to 0.98 . Knowledge of all virial coefficients up to $B_{M}$ was used, higher were predicted. The error bounds in parentheses include both systematic and statistical contributions.

\begin{tabular}{|c|c|c|c|c|c|c|c|}
\hline$n$ & $M=4$ & $M=5$ & $M=6$ & $M=7$ & $M=8^{\dagger}$ & $M=9$ & $M=10$ \\
\hline \hline 5 & $28.255(30)$ & & & & & & \\
6 & $39.67(7)$ & $39.716(23)$ & & & & & \\
7 & $53.08(23)$ & $53.314(20)$ & $53.31(12)$ & & & & \\
8 & $69.0(2)$ & $69.36(9)$ & $68.6(2)$ & $68.66(5)$ & & & \\
9 & $87.7(10)$ & $87.6(5)$ & $85.8(5)$ & $85.74(14)$ & $85.30(8)$ & & \\
10 & $109(3)$ & $106.8(8)$ & $105.9(18)$ & $104.9(2)$ & $105.1(5)$ & $106.3(1)$ & \\
11 & $130(5)$ & $126.0(9)$ & $130.3(40)$ & $128(2)$ & $130.5(7)$ & $130.9(7)$ & $130.5(10)$ \\
12 & $150(3)$ & $142(2)$ & $160(5)$ & $158(7)$ & $164.2(9)$ & $156(3)$ & $156(3)$ \\
13 & $162(15)$ & $152(6)$ & $190(10)$ & $197(11)$ & $204(4)$ & $174(9)$ & $176(4)$ \\
14 & $160(40)$ & $159(20)$ & $230(40)$ & $230(15)$ & $232(26)$ & $175(10)$ & $184(8)$ \\
15 & $130(70)$ & $161(30)$ & $180(100)$ & $240(20)$ & $210(40)$ & $165(50)$ & $179(32)$ \\
\hline
\end{tabular}

${ }^{\dagger}$ see figure 1 


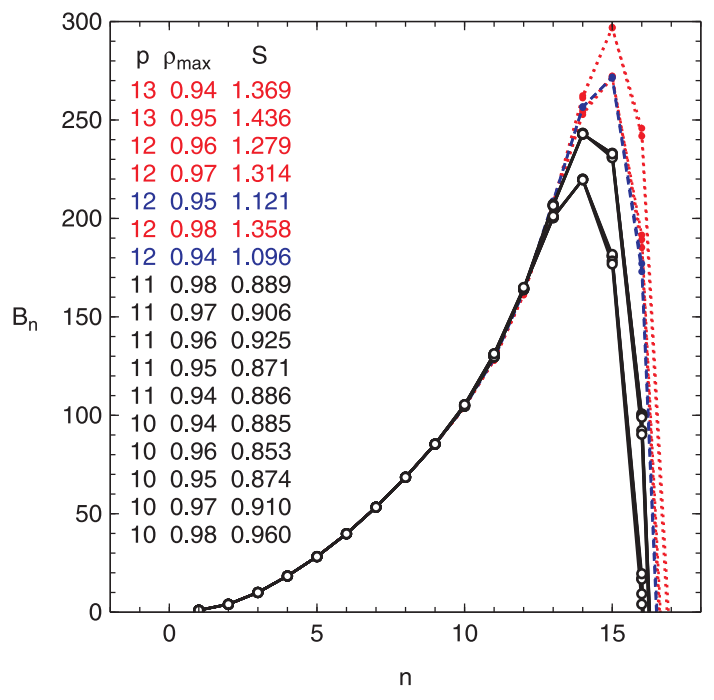

Figure 1. (Color online) Example of extrapolating the virial coefficients using formulas $Z=1+\sum_{i=1}^{9} a_{i} x^{i}+$ $a_{p} x^{p}$ based on $B_{2}$ to $B_{8}$ and EOS data up to $\rho_{\max }$. Virial coefficients $B_{n}, n>8$, are predicted, see the marked $^{\dagger}$ column of table 3 Black solid lines are the most precise fits $(S<1)$, blue dashed lines are less precise $(S \in[1,1.2))$, and red dotted lines the least precise $(S \in[1.2,1.5))$. The table is ordered according to the predicted $B_{15}$ value and color-coded in the same way as the lines; some lines overlap.

Table 4. The same as table 3 with expansion [2.4.

\begin{tabular}{|c|c|c|c|c|c|c|c|}
\hline$n$ & $M=4$ & $M=5$ & $M=6$ & $M=7$ & $M=8^{\dagger}$ & $M=9$ & $M=10$ \\
\hline \hline 5 & $28.240(35)$ & & & & & & \\
6 & $39.73(11)$ & $39.72(2)$ & & & & & \\
7 & $53.2(3)$ & $53.300(13)$ & $53.26(3)$ & & & & \\
8 & $69.1(3)$ & $69.24(7)$ & $68.50(3)$ & $68.67(3)$ & & & \\
9 & $87.3(14)$ & $87.4(4)$ & $86.0(3)$ & $85.66(8)$ & $85.48(9)$ & & \\
10 & $107(4)$ & $107.0(7)$ & $106.4(7)$ & $104.80(16)$ & $105.3(3)$ & $106.15(6)$ & \\
11 & $129(5)$ & $126.7(5)$ & $131.2(4)$ & $127.7(6)$ & $130.38(12)$ & $130.8(2)$ & $130.6(3)$ \\
12 & $148(9)$ & $144.7(8)$ & $162(3)$ & $157(4)$ & $162.2(24)$ & $158.1(14)$ & $158(2)$ \\
13 & $164(9)$ & $158(4)$ & $192(6)$ & $193(8)$ & $205(13)$ & $182(4)$ & $182(3)$ \\
14 & $174(35)$ & $165(12)$ & $207(16)$ & $227(11)$ & $234(35)$ & $192(7)$ & $192(4)$ \\
15 & $170(120)$ & $145(45)$ & $175(20)$ & $240(10)$ & $200(15)$ & $170(20)$ & $171(8)$ \\
\hline
\end{tabular}

† see figure1

The number of adjustable parameters is important in obtaining relevant predictions. We started with a small set and increased the number of adjustable parameters until the value of $S$ dropped below 1.5. However, we never used more parameters if $S<1$ was already reached to avoid a fitting noise. For each set of virial coefficients up to $M, 4 \leqslant M \leqslant 10$, we analyzed several maximum powers in expansion (2.3) with all the above mentioned EOS data sets. The results were sorted into three categories: the best with $S<1$, the good ones with $S<1.2$, and the worst results with $S<1.5$. Then, we plotted the curves of the predicted $B_{n}$ vs. $n$ and graphically determined the median value and also tried to estimate the uncertainty from the data scattering. This error estimate is rather sensitive to the systematic errors which are larger than the statistical ones. We admit a subjective factor present in this procedure. One example is shown in figure 1 .

The results, based on more than a thousand of equations analyzed, are collected in tables 3 and 4 It is seen that the procedure is capable of predicting the virial coefficients even though the errors are 
sometimes underestimated. Of course, the virial coefficients obtained are less accurate than the directly obtained MC values. Only for $B_{10}$, the precision of the predicted value based on all previous coefficients approaches the precision of the MC result [7, 8]. In addition, the accuracy of $B_{14}$ and especially $B_{15}$ decreases.

\subsection{Padé approximants}

Simultaneous fitting of MD data and virial coefficients to rational functions (Padé approximants) is much more difficult because the equations are highly nonlinear. The approximants often give oscillating solutions from $B_{12}$ or $B_{13}$; we omit these solutions because we assume that the coefficients should behave "regularly".

First we investigated the rational functions using all the known information, i.e., the exact values of $B_{2}$ to $B_{4}$, approximate values of $B_{5}$ to $B_{10}$ with standard deviations, and MD data to $\rho_{\max }=0.94,0.95,0.96$, 0.97, 0.98 (five tested variants). Approximants Pade [2,12], [8,5], [3,10], and [9,3] are the best (interestingly, [9,3] requires by 2 less parameters than [2,12]; [2,11] is worse, $S=1.4$, pushing $B_{14}$ to lower values) which with the five maximum densities gives 20 curves from which the predictions and their errors were determined. To test the predictive power of the procedure, we repeated the calculations without knowledge of $B_{7}$ to $B_{10}$, see table 5. It is seen that the Padé approximants predict worse values of the lower virial coefficients than the Barboy and Gelbart approach. Nevertheless, the predictions of $B_{11}$ to $B_{15}$ using all the data are comparable.

Table 5. Predictions using Padé approximants. See table 3 for the explanation of symbols.

\begin{tabular}{|c|c|c|}
\hline$n$ & $M=6$ & $M=10$ \\
\hline \hline 7 & $53.28(7)$ & \\
8 & $68.94(16)$ & \\
9 & $84.7(14)$ & \\
10 & $104.0(13)$ & \\
11 & $130.6(4)$ & $130.5(10)$ \\
12 & $162(15)$ & $158(3)$ \\
13 & $195(30)$ & $181(4)$ \\
14 & $200(20)$ & $196(10)$ \\
15 & $150(60)$ & $198(24)$ \\
\hline
\end{tabular}

\subsection{Reliability of extrapolations}

While the statistical errors in the predicted higher virial coefficients can be easily evaluated, this cannot be said about the systematic errors introduced by a particular functional form. The only way to assess the systematic errors is to compare as many functional forms (Padé indices, powers in polynomials, etc.) as possible. Then, we subjectively estimate (after cancelling the evidently defective values) the "most probable" values of them.

An example of a comparison of different methods of extrapolation is shown in table 6 for the fourteenth virial coefficient. In the table, ten particular extrapolation results are shown: three utilizing the Barboy-Gelbart equation (2.3) (with and without adjustable virial coefficients), Padé approximants, and a differential approximant, equation (2.2).

The final recommended values of higher virial coefficients including the estimated uncertainties are given in table 7 They are based on critical examination of several methods and many equations of state. In the table, our values are compared with recent literature estimates obtained independently using different approaches by different authors [7, 8, 26-29]. Except $B_{15}$ they agree with ours within estimated errors. However, a general trend is that we predict $B_{11}$ to $B_{13}$ higher whereas $B_{14}$ and $B_{15}$ lower.

In the table our estimated values of $B_{n}$ are shown up to $n=15$. We calculated higher virial coefficients as well. However, their values exhibited a large scatter and no final estimates were thus made. Anyway, the literature estimates for $B_{16}$ are given in the table. 
Table 6. The extrapolated fourteenth virial coefficient using all the known virial coefficients and densities up to $\rho=0.94$. BG+1 denotes the Barboy-Gelbart equation (2.3) with one additional term $x^{10}$, BG+11 with $x^{10}$ and $x^{11}$, etc., and no optimization of the virial coefficients values, $\mathrm{O}$ - in front of symbols means that the full optimization, [2.5), was performed. Padé and O-Padé denote the nonoptimized and optimized Padé approximants, equation [2.1. O-DA is the optimized differential approximant.

\begin{tabular}{|l|r|r|}
\hline Method & $B_{14}$ & $S$ \\
\hline \hline BG+1 & -270 & 234 \\
BG+11 & 55 & 19 \\
BG+111 & 198 & 1.54 \\
O-BG+1 & 215 & 1.59 \\
O-BG+11 & 193 & 1.45 \\
O-BG+111 & 186 & 0.97 \\
Pade [5,4] & 204 & 8.3 \\
Pade [2,7] & 198 & 11.3 \\
O-Pade [5,4] & 207 & 6.9 \\
O-Pade [2,7] & 206 & 6.7 \\
O-Pade [9,1] & 204 & 6.5 \\
O-Pade [7,3] & 201 & 6.3 \\
O-DA & 200 & 6.3 \\
\hline
\end{tabular}

Table 7. Extrapolated virial coefficients.

\begin{tabular}{|l|l|l|l|l|l|l|}
\hline$n$ & this work & ref. [7, 8] & ref. [26] & ref. [27] & ref. [28] & ref. [29] \\
\hline \hline 11 & $\mathbf{1 3 0 . 6 ( 1 0 )}$ & 128 & 128 & 129 & 126 & 128 \\
12 & $\mathbf{1 5 7 ( 3 )}$ & 153 & 153 & 154 & 149 & 153 \\
13 & $\mathbf{1 8 5 ( 1 0 )}$ & 181 & 181 & 182 & 174 & 182 \\
14 & $\mathbf{1 9 0 ( 2 0 )}$ & 215 & 213 & 211 & 202 & 214 \\
15 & $\mathbf{1 8 0 ( 3 0 )}$ & 247 & 248 & 236 & 231 & 247 \\
16 & - & 279 & 288 & 254 & 262 & 279 \\
\hline
\end{tabular}

We cannot assert that really $B_{15}<B_{14}$; in fact, oscillating behavior is typical of polynomial approximants and these results are consistent with a monotonous increase, at least for not too large $n$. Ultimately the sequence of $B_{n}$ cannot be monotonous because the equation of state is not analytical at the freezing point and consequently its radius of convergence cannot exceed the freezing density (we believe that it equals the freezing density).

\section{Concluding remarks}

In this work we have used several extrapolation methods to estimate the unknown virial coefficients of hard spheres from $B_{11}$ to $B_{15}$ and compared them with recent literature estimates. We believe that the presented results could be used in testing theoretical approaches of the thermodynamics of hard spheres.

The problem of reliability of any extrapolation result rests in the estimate of its uncertainty. This is, in principle, subjective depending on an author's view (either optimistic or pessimistic). Here we have tried neither to be too optimistic nor too pessimistic. We have used comparisons of extrapolated virial coefficients obtained using as many different approaches as possible.

The techniques used in this work can be readily extended to virial coefficients of hard body fluids such as spherocylinders, diatomics and multi-atomics, mixtures of hard spheres and other hard body fluids and their mixtures. What one needs is i) reliable values of lower virial coefficients and estimates of their uncertainties, and ii) precise equation-of-state computer simulation data. An extrapolation of virial coefficients of non-hard-body fluids is less straightforward due to their dependence on temperature. 


\title{
References
}

1. Hill T.L., Statistical Mechanics, McGraw-Hill, New York, 1956.

2. Nijboer B.R.A., Van Hove L., Phys. Rev., 1952, 85, 777; doi 10.1103/PhysRev.85.777

3. Lyberg I., e-print cond-mat/0410080.

4. Ree F.H., Hoover W.G., J. Chem. Phys., 1964, 40, 939; doi 10.1063/1.1725286.

5. Vlasov A.Y., You X.-M., Masters A.J., Mol. Phys., 2002, 100, 3313; doi 10.1080/00268970210153754

6. Labík S., Kolafa J., Malijevský A., Phys. Rev. E, 2005, 71, 021105; doi 10.1103/PhysRevE.71.021105

7. Clisby N., McCoy B.M., J. Stat. Phys., 2006, 122, 15; doi 10.1007/s10955-005-8080-0

8. Clisby N., McCoy B.M., Pramana-J. Phys., 2005, 64, 775; doi 10.1007/BF02704582

9. Allen M.P., Tildesley D.J., Computer Simulation of Liquids. Clarendon Press, Oxford, 1987.

10. Rosenbluth M.N., Rosenbluth A.W., J. Chem. Phys., 1955, 23, 356; doi 10.1063/1.1741967.

11. Kratky K.W., Physica A, 1976, 85, 607; doi 10.1016/0378-4371(76)90029-7

12. Kratky K.W., Physica A, 1977, 87, 584; doi 10.1016/0378-4371(77)90051-6

13. Kratky K.W., J. Stat. Phys., 1982, 27, 533; doi 10.1007/BF01011091

14. Kratky K.W., J. Stat. Phys., 1982 29, 129; doi 10.1007/BF01008253

15. Ree F.H., Hoover W.G., J. Chem. Phys., 1967, 46, 4181; doi 10.1063/1.1840521

16. Kim S., Henderson D., Phys. Lett. A, 1968, 27, 378; doi 10.1016/0375-9601(68)91066-9

17. van Rensburg E.J., Torrie G.M., J. Phys. A: Math. Gen., 1993, 26, 943; doi 10.1088/0305-4470/26/4/022

18. van Rensburg E.J., J. Phys. A: Math. Gen., 1993, 26, 4805; doi 10.1088/0305-4470/26/19/014

19. Sanchez I.C., J. Chem. Phys., 1994, 101, 7003; doi 10.1063/1.468456

20. Guttmann A.J. - In: Phase Transitions and Critical Phenomena, Vol. 3, chapter 1, eds. C. Domb and J. Lebowitz. Academic Press, 1989.

21. Kolafa J., Labík S., Malijevský A., Phys. Chem. Chem. Phys., 2004, 6, 2335; doi 10.1039/b402792b

22. Barboy B., Gelbart W.M., J. Chem. Phys., 1979, 71, 3053; doi 10.1063/1.438711.

23. Yelash L.V., Kraska T., Phys. Chem. Chem. Phys., 2001, 3, 3114; doi 10.1039/b102972j

24. Erpenbeck J.J., Wood W.W., J. Stat. Phys., 1984, 35, 321; doi 10.1007/BF01014387

25. Kolafa J., Phys. Chem. Chem. Phys., 2006, 8, 464; doi 10.1039/b511999e

26. Tian J.X., Gui Y.X., Mulerlo A., Phys. Chem. Chem. Phys., 2009, 11, 11213; doi 10.1039/b915002a

27. Bannerman M.N., Lue L., Woodcock L.V., J. Chem. Phys., 2010, 132, 084507; doi 10.1063/1.3328823

28. Hu J.W., Yu Y.X., Phys. Chem. Chem. Phys., 2009, 11, 9382; doi 10.1039/b911901a

29. Tian J.X., Gui Y.X., Mulerlo A., J. Phys. Chem. B, 2010, 114, 13399; doi 10.1021/jp106502x

\section{До екстраполяції віріальних коефіцієнтів твердих сфер}

\author{
М. Ончак ${ }^{1}$, А. Малієвск 112, Й. Колафа $a^{11}$, С. Лабік ${ }^{11}$ \\ 1 Інститут хімічної технології, Прага, Відділ фізичної хімії, 16628 Прага 6, Чеська Республіка \\ 2 Відділ фізики, факультет природничих наук, університет м. Острава, 70103 Острава 1, Чеська Республіка
}

Обговорюються декілька екстаполяційних методів для віріальних коефіцієнтів, включно із запропонованими в цій роботі. Показано можливості методів передбачати вищі віріальні коефіцієнти однокомпонентної системи твердих сфер. Запропоновано оцінки для віріальних коефіцієнтів від одинадцятого до п'ятнадцятого. Передбачається, що віріальні коефіцієнти $B_{n}$ вищого порядку ніж $B_{14}$, можуть зменшуватися з ростом $n$ і можуть досягати негативних значень для великих $n$. Ці екстраполяційні методи можуть бути використані в інших областях, де екстраполяція $є$ важливою.

Ключові слова: тверді сфери, віріальні коефіцієнти, екстраполяційні методи 